\title{
Antioxidant capacities of fucoxanthin-producing algae as influenced by their carotenoid and phenolic contents
}

\begin{abstract}
Natural antioxidants from sustainable sources are favoured to accommodate worldwide antioxidant demand. In addition to bioprospecting for natural and sustainable antioxidant sources, this study aimed to investigate the relationship between the bioactives (i.e. carotenoid and phenolic acids) and the antioxidant capacities in fucoxanthin-producing algae. Total carotenoid, phenolic acid, fucoxanthin contents and fatty acid profile of six species of algae (five microalgae and one macroalga) were quantified followed by bioactivity evaluation using four antioxidant assays. Chaetoceros calcitrans and Isochrysis galbana displayed the highest antioxidant activity, followed by Odontella sinensis and Skeletonema costatum which showed moderate bioactivities. Phaeodactylum tricornutum and Saccharina japonica exhibited the least antioxidant activities amongst the algae species examined. Pearson correlation and multiple linear regression showed that both carotenoids and phenolic acids were significantly correlated $(\mathrm{p}<0.05)$ with the antioxidant activities, indicating the influence of these bioactives on the algal antioxidant capacities.
\end{abstract}

Keyword: Microalgae mass culture; Antioxidant activity; Carotenoid; Fucoxanthin; Phenolic acids; PUFA profiles 